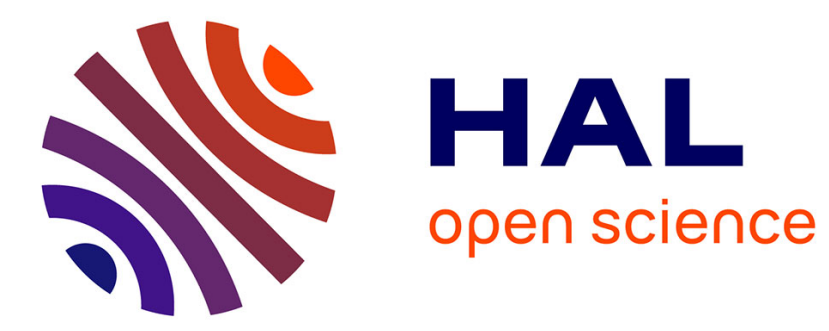

\title{
Opinion propagation in bounded medium-sized populations
}

Eline de Cuypere, Koen de Turck, Sabine Wittevrongel, Dieter Fiems

\section{To cite this version:}

Eline de Cuypere, Koen de Turck, Sabine Wittevrongel, Dieter Fiems. Opinion propagation in bounded medium-sized populations. Performance Evaluation, 2016, 99, pp.1 - 15. 10.1016/j.peva.2016.03.001 . hal-01426179

\section{HAL Id: hal-01426179 \\ https://hal.science/hal-01426179}

Submitted on 4 Jan 2017

HAL is a multi-disciplinary open access archive for the deposit and dissemination of scientific research documents, whether they are published or not. The documents may come from teaching and research institutions in France or abroad, or from public or private research centers.
L'archive ouverte pluridisciplinaire HAL, est destinée au dépôt et à la diffusion de documents scientifiques de niveau recherche, publiés ou non, émanant des établissements d'enseignement et de recherche français ou étrangers, des laboratoires publics ou privés. 


\title{
Opinion propagation in bounded medium-sized populations
}

\author{
Eline De Cuypere ${ }^{1}$, Koen De Turck ${ }^{2}$, Sabine Wittevrongel ${ }^{1}$ and Dieter Fiems ${ }^{1}$ \\ 1 Department of Telecommunications and Information Processing, Ghent University, \\ St-Pietersnieuwstraat 41, B-9000 Gent, Belgium \\ 2 Laboratoire des Signaux 8 Systèmes, Centrale Supélec, \\ 3, Rue Joliot-Curie, 91192 Gif sur Yvette, France
}

\begin{abstract}
We study the dynamics of opinion propagation in a medium-sized population with low population turnover. Opinion spreading is modelled by a Markovian non-standard Susceptible-Infected-Recovered (SIR) epidemic model with stochastic arrivals, departures, infections and recoveries. The system performance is evaluated by two complementary approaches: a numerical but approximate solution approach which relies on Maclaurinseries expansions of the stationary solution of the Markov process and a fluid limit approach. Both methods are evaluated numerically. Moreover, convergence to the fluid limit is proved, and explicit expressions for the fixed points of the differential equations are obtained for the case of linearly increasing infection and arrival rates.
\end{abstract}

Keywords: Opinion propagation; Epidemic model; Maclaurin-series expansion; Fluid model; Markov chain; Performance analysis.

\section{Introduction}

Given the rapid growth of companies in the internet sector that base their revenue model on advertisement (such as Google, Facebook, etc.) [16] and the ascent of social networks in particular, the study of opinion spreading is a trending topic, and there is a strong interest in understanding how new opinions spread through a community. Apart from these economic considerations, the analysis of opinion spreading can improve our comprehension of social relations among individuals, both online and offline.

This paper studies opinion propagation by drawing parallels with the spreading of diseases $[4,19]$. Indeed, opinion propagation bears some similarity to the spread of an infectious disease, and particularly to Kermack and McKendrick's classical compartmental SIR model for such propagation [26]. The acronym SIR stands for susceptible $(\mathrm{S})$, infectious $(\mathrm{I})$ and recovered $(\mathrm{R})$, and refers to the possible states that an individual can be in, the possible transitions between these states following the order $\mathrm{S} \rightarrow \mathrm{I} \rightarrow \mathrm{R}$. In particular, the SIR model assumes that if a healthy individual encounters an infected individual, there is a chance that the healthy individual gets infected. An infected individual then recovers from the disease after some time, making him/her immune for the infection. This process can be directly reformulated in terms of the 
propagation of opinions on a particular topic: a susceptible or non-opinionated individual has yet to form an opinion on the topic, whereas infected or opinionated individuals do have such an opinion. Susceptible individuals may form an opinion when they encounter infected individuals, like an infection is carried over to a healthy individual upon contact. Finally, individuals loose their interest in the topic after some time and stop spreading their opinion. These individuals have recovered.

The SIR epidemic model has been predominantly applied to study disease, see e.g. [19, 20, 21, 23], but there is some prior work in the areas of rumour and opinion propagation as well. Concerning the latter, Zhao extends the SIR model for opinion spreading in social networks by including a hibernator state in which individuals temporary interrupt infecting others [39]. Moreno et. al study SIR like spreading of rumours explicitly accounting for the network structure [31]. Bettencourt et. al [5] draw parallels between epidemics and idea diffusion by applying several epidemiological models to empirical data. Woo et. al [37] show the plausibility to describe the mechanism of violent topic diffusion in web forums by a SIR model while Fan et. al [17] propose an extended SIR model for opinion dynamics in which individuals can have a positive or negative opinion about a topic. Finally, some preliminary results on series expansion techniques (cf. infra) for stochastic SIR models for opinion propagation were presented in [12].

SIR models are not the only models for studying opinion propagation in literature. For example, the threshold model starts from a random directed graph where each node selects a random threshold [25]. Opinion propagation then evolves deterministically: a node becomes active (gets an opinion) if the fraction of its active neighbours exceeds its threshold. In contrast to the threshold model, the dynamics of the voter model are stochastic: each node changes its state to the state of a random neighbour [28]. The Sznajd model [36] assumes more complex interactions between nodes: a node and a neighbouring node are selected at random. If the neighbouring node is undecided, it adopts the opinion with some probability. If both have the same opinion, they try to convince the other neighbours. Finally, if they have different opinions, nothing happens. Specifically focussing on tweet propagation, Yang et. al [38] propose a factor graph model based on the analysis of the factors influencing the user's retweet behaviour. Kawamoto et. al [24] model the information diffusion as a random multiplicative process, with a particular focus on retweet behaviour. In [22], the traditional Susceptible-Infected-Susceptible (SIS) epidemic model is studied in order to predict retweeting trends.

In this paper, we focus on a compartmental Markovian SIR model for opinion spreading in bounded medium-sized populations. While the total population of internet users easily qualifies as large, the size of an online community - say, of people contributing to an online forum or of people tweeting and retweeting some hash tag - is often not that large. Moreover, these communities hardly remain constant over time, with individuals joining and leaving all the time. We study such communities assuming that the number of individuals is bounded, there is a maximum number of individuals in the community. Such epidemic processes on bounded medium-sized populations are interesting from a mathematical point of view as well. 
While our numerical results reveal that a fluid limit approach yields accurate results when individuals only remain for a limited time (cf. figure 7), such an approach cannot be used to assess the system when individuals remain for a long time. For small populations, this is not problematic as small population sizes translate into small state spaces of the corresponding Markov processes such that the steady-state probability vector is easily calculated. In contrast, when the bound (and therefore also the state space) is larger - we use the term medium-sized populations - direct calculation of the stationary vector is computationally infeasible and performance needs to be assessed by other means when individuals remain for a long time. This is the subject of the present paper.

The contributions of this paper are twofold. First, we investigate the performance of opinion propagation in a Markovian framework by an approximate solution technique for Markov processes which relies on Maclaurin-series expansions of the steady-state probability vector. This technique was recently applied to study kitting processes [13]; a kitting process is a type of multi-buffer queueing system in which service is synchronised between the different buffers and temporarily blocked if one of the buffers is empty [11]. The epidemic process under consideration generalises the Markovian SIR process in various ways. We assume that the population size is bounded, but individuals join and leave the population over time to account for the dynamic formation of online communities. Moreover, the assumptions on infection and recovery rates are relaxed and individuals are allowed to move from susceptible to recovered directly as community members not necessarily want to spread the opinion to others. Secondly, in addition to the Maclaurin-series approach, we consider a fluid limit of the Markov process at hand and formally prove convergence. Fluid limits are a popular mathematical technique (see e.g. [8, 9, 14, 15, 29, 33, 34] and the references therein) which (when a good scaling is found) allow for focussing on the salient features of the stochastic process while discarding 'second-order fluctuations' around this main trend. In the present paper, it helps to make the link with more standard deterministic SIR models. We like to mention that the fluid scaling under study (arrival rates and location capacity are sent to infinity), differs significantly and therefore complements the Maclaurinseries expansion limit (which holds for low departure rates). We thus aim to view this computationally cumbersome Markov model from different limiting cases, and gain new insights by combining them. We also note that the derivation of the fluid limit as performed in this paper also lends itself naturally to refinements in the form of diffusion results, but this is considered to be outside of the scope of the current paper.

The remainder of this paper is organised as follows. Section 2 introduces the opinion spreading model at hand as well as some particular examples, discussed further on. In section 3 , the balance equations are derived and the numerical series expansion approach is explained. Next, we find a fluid limit for the epidemic Markov process in section 4 . To illustrate both approaches, section 5 considers various numerical examples. Finally, conclusions are drawn in section 6 . 


\section{Model description}

We consider an opinion propagation system as depicted in Figure 1. There are at most $L$ individuals in the community, each individual either being recovered (r), infected (i) or susceptible (s) (this particular ordering instead of the traditional $s, i, r$ will prove useful for the Maclaurin analysis of section 3$)$. Let $X_{k}(t)$ be the number of individuals of type $k \in \mathcal{K}=\{r, i, s\}$ at time $t$, and let $\mathbf{X}(t)=\left(X_{r}(t), X_{i}(t), X_{s}(t)\right) \in \mathcal{L}=$ $\left\{\left(x_{r}, x_{i}, x_{s}\right) \in \mathbb{N}^{3} \mid x_{i}+x_{r}+x_{s} \leq L\right\}$. For any $\mathbf{x} \in \mathcal{L}, x_{k}$ is the number of individuals of type $k \in \mathcal{K}$ and $\|\mathbf{x}\|_{1}=\left|x_{r}\right|+\left|x_{i}\right|+\left|x_{s}\right|=x_{r}+x_{i}+x_{s}$ is the $L_{1}$ norm which corresponds to the total number of individuals.

We consider a Markovian opinion propagation system, the number of individuals of the different types being the state of the Markov process. We make the following assumptions on the arrival, infection and recovery rates of the Markov process.

- For, $\|\mathbf{X}(t)\|_{1}<L$, there is a new arrival of type $k \in \mathcal{K}$ in the interval $[t, t+d t)$ with probability $\lambda_{k}(\mathbf{X}(t)) d t+o(d t)$. The total arrival rate in state $\mathbf{x} \in \mathcal{L}$ is denoted by $\lambda(\mathbf{x})=\lambda_{r}(\mathbf{x})+\lambda_{i}(\mathbf{x})+\lambda_{s}(\mathbf{x})$. To simplify notation, assume $\lambda(\mathbf{x})=\lambda_{k}(\mathbf{x})=0$ for $\|\mathbf{x}\|_{1} \geq L$ and $k \in \mathcal{K}$. The dependence of the arrival rate on the population size is motivated by the observation that having many individuals in a community can be seen as a quality signal which then results in more individuals joining the community (cf. section 2.2 infra).

- There is a departure of an individual of type $k \in \mathcal{K}$ in the interval $[t, t+d t)$ with probability $\mu X_{k}(t) d t+$ $o(d t)$. Hence, the residence time of any individual is exponentially distributed with mean $1 / \mu$.

- A single susceptible (infected, susceptible) individual gets infected (recovers, recovers, respectively) in the interval $[t, t+d t)$ with probability $\alpha_{s i}(\mathbf{X}(t)) d t+o(d t)\left(\alpha_{i r}(\mathbf{X}(t)) d t+o(d t), \alpha_{s r}(\mathbf{X}(t)) d t+o(d t)\right)$. To simplify further notation, we assume $\alpha_{s i}(\mathbf{x})=\alpha_{s r}(\mathbf{x})=0$ for $x_{s}=0$ and $\alpha_{i r}(\mathbf{x})=0$ for $x_{i}=0$. There can be no infection or recovery if there are no individuals that can get infected or that can recover.

The Maclaurin-series expansion in section 3 further requires that for every $\mathbf{x} \in \mathcal{L}$, (i) the total arrival rate $\lambda(\mathbf{x})$ is non-zero, (ii) the infection rate $\alpha_{s i}(\mathbf{x})$ and the refusing rate $\alpha_{s r}(\mathbf{x})$ are non-zero for $x_{s}>0$, and (iii) the recovery rate $\alpha_{i r}(\mathbf{x})$ is non-zero for $x_{i}>0$.

Let $\pi(\cdot)$ denote the stationary distribution of the Markov chain (which is guaranteed to exist as the state space is finite and uni-chain), and - for further use - let $\mathbf{X}$ denote a generic random variable distributed 


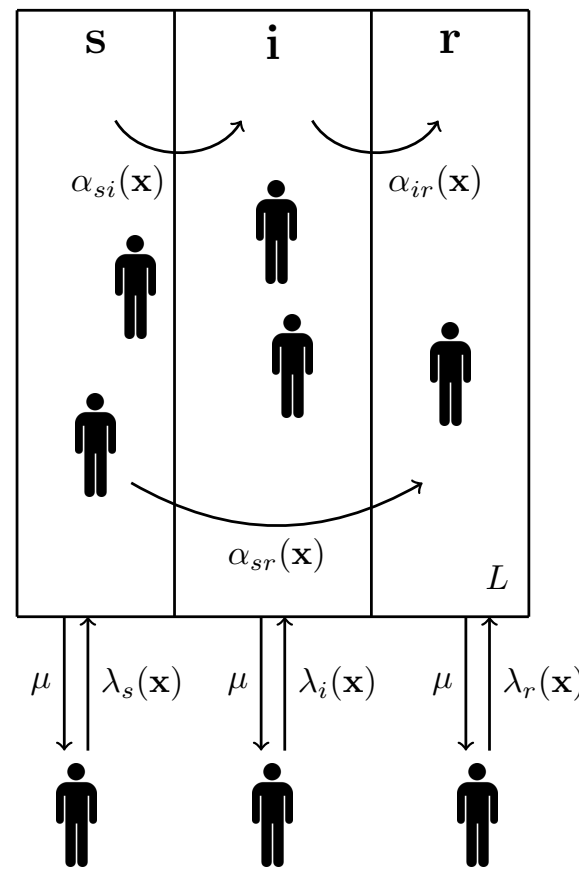

Figure 1: Opinion propagation model.

according to $\pi$. From the above generator representation we derive the following set of balance equations:

$$
\begin{aligned}
\pi(\mathbf{x})\left(\|\mathbf{x}\|_{1} \mu\right. & \left.+\lambda(\mathbf{x})+\alpha_{s i}(\mathbf{x})+\alpha_{i r}(\mathbf{x})+\alpha_{s r}(\mathbf{x})\right) \\
= & \pi\left(x_{r}+1, x_{i}, x_{s}\right) \mu\left(x_{r}+1\right)+\pi\left(x_{r}, x_{i}+1, x_{s}\right) \mu\left(x_{i}+1\right)+\pi\left(x_{r}, x_{i}, x_{s}+1\right) \mu\left(x_{s}+1\right) \\
& \quad+\pi\left(x_{r}-1, x_{i}, x_{s}\right) \lambda_{r}\left(x_{r}-1, x_{i}, x_{s}\right)+\pi\left(x_{r}, x_{i}-1, x_{s}\right) \lambda_{i}\left(x_{r}, x_{i}-1, x_{s}\right) \\
& \quad+\pi\left(x_{r}, x_{i}, x_{s}-1\right) \lambda_{s}\left(x_{r}, x_{i}, x_{s}-1\right)+\pi\left(x_{r}, x_{i}-1, x_{s}+1\right) \alpha_{s i}\left(x_{r}, x_{i}-1, x_{s}+1\right) \\
& +\pi\left(x_{r}-1, x_{i}+1, x_{s}\right) \alpha_{i r}\left(x_{r}-1, x_{i}+1, x_{s}\right)+\pi\left(x_{r}-1, x_{i}, x_{s}+1\right) \alpha_{s r}\left(x_{r}-1, x_{i}, x_{s}+1\right)
\end{aligned}
$$

for $\mathbf{x} \in \mathcal{L}$. Here and in the remainder, we follow the convention that $\pi(\mathbf{x})=0$ if $\mathbf{x} \notin \mathcal{L}$.

Prior to introducing the series expansions and fluid approximations, we first introduce two particular examples of the Markov process. In section 5 where we present various numerical results, we return to these examples.

\subsection{Constant infection and arrival rates}

In the most basic setting, individuals arrive according to a Poisson process with the (state-independent) parameter $\lambda_{k}, k \in \mathcal{K}$. Furthermore, if we assume that each susceptible individual has a constant probability to get infected and to recover, i.e. $\alpha_{s i}(\mathbf{x})=x_{s} \alpha_{s i}$ and $\alpha_{s r}(\mathbf{x})=x_{s} \alpha_{s r}$, and that each infected individual has 
a constant probability to recover, i.e. $\alpha_{i r}(\mathbf{x})=x_{i} \alpha_{i r}$, then the mean number of each type in steady state can be calculated explicitly.

Indeed, as the departure rate of each individual is equal to $\mu$, the total number of individuals $\|\mathbf{X}\|_{1}$ is distributed as the queue content of a classic $M / M / L / L$ queue, with arrival rate $\lambda=\lambda_{r}+\lambda_{i}+\lambda_{s}$ and departure rate $\mu$, for which the steady-state distribution can be found in every queueing-theory textbook,

$$
\operatorname{Pr}\left[\|\mathbf{X}\|_{1}=n\right]=\frac{\frac{1}{n !}\left(\frac{\lambda}{\mu}\right)^{n}}{\sum_{m=0}^{L} \frac{1}{m !}\left(\frac{\lambda}{\mu}\right)^{m}} .
$$

Let $p_{k}$ denote the probability that a random departing individual is of type $k \in \mathcal{K}$. An individual leaves the system as a susceptible individual, provided it arrived in the system as a susceptible individual and it leaves the system prior to infection or recovery. Hence, we have,

$$
p_{s}=\frac{\lambda_{s}}{\lambda} \frac{\mu}{\alpha_{s i}+\mu+\alpha_{s r}},
$$

the first factor being the probability that an arriving individual is susceptible and the second factor being the probability that a susceptible individual leaves prior to infection or recovery. Analogously, an individual departs as an infected individual if (i) it arrives as susceptible, gets infected and does not recover or (ii) it arrives as infected and does not recover. Hence, we find,

$$
p_{i}=\frac{\lambda_{i}}{\lambda} \frac{\mu}{\alpha_{i r}+\mu}+\frac{\lambda_{s}}{\lambda} \frac{\alpha_{s i}}{\left(\alpha_{s i}+\alpha_{s r}+\mu\right)} \frac{\mu}{\left(\alpha_{i r}+\mu\right)} .
$$

Finally, an individual leaves as recovered if it does not leave the system as a susceptible or infected individual, hence we have,

$$
p_{r}=1-p_{i}-p_{s}=1-\frac{\lambda_{s}}{\lambda} \frac{\mu}{\alpha_{s i}+\mu+\alpha_{s r}}-\frac{\lambda_{i}}{\lambda} \frac{\mu}{\alpha_{i r}+\mu}-\frac{\lambda_{s}}{\lambda} \frac{\alpha_{s i}}{\left(\alpha_{s i}+\alpha_{s r}+\mu\right)} \frac{\mu}{\left(\alpha_{i r}+\mu\right)} .
$$

Note that the rate at which an individual leaves the system does not depend on the type of the individual. Therefore $p_{k}$ is the probability that a random individual in the system is of type $k$. Moreover, individuals change type, independent of other individuals. Hence, the distribution of the number of individuals of the different types, conditioned on the total number of individuals in the system, is a multinomial distribution with parameters $p_{r}, p_{i}$ and $p_{s}$. Combining this observation with equation (2), yields,

$$
\operatorname{Pr}[\mathbf{X}=\mathbf{x}]=\operatorname{Pr}\left[\|\mathbf{X}\|_{1}=\|\mathbf{x}\|_{1}\right] \frac{\|\mathbf{x}\|_{1} !}{x_{r} ! x_{i} ! x_{s} !} p_{r}^{x_{r}} p_{i}^{x_{i}} p_{s}^{x_{s}}=\left(\frac{\lambda}{\mu}\right)^{\|\mathbf{x}\|_{1}} \frac{p_{r}^{x_{r}} p_{i}^{x_{i}} p_{s}^{x_{s}}}{x_{r} ! x_{i} ! x_{s} !}\left(\sum_{m=0}^{L} \frac{1}{m !}\left(\frac{\lambda}{\mu}\right)^{m}\right)^{-1}
$$

and,

$$
\operatorname{Pr}\left[X_{k}=n\right]=\sum_{\ell=n}^{L}\left(\begin{array}{l}
\ell \\
n
\end{array}\right) \operatorname{Pr}\left[\|\mathbf{X}\|_{1}=\ell\right] p_{k}^{n}\left(1-p_{k}\right)^{\ell-n}=\sum_{\ell=n}^{L}\left(\frac{\lambda}{\mu}\right)^{\ell} \frac{p_{k}^{n}}{n !} \frac{\left(1-p_{k}\right)^{\ell-n}}{(\ell-n) !}\left(\sum_{m=0}^{L} \frac{1}{m !}\left(\frac{\lambda}{\mu}\right)^{m}\right)^{-1} .
$$




\subsection{A model for opinion spreading}

The following example is more complex and does not have a simple solution. We will rely on either a Maclaurin-series approach or on a fluid limit to estimate performance.

We propose the following infection and recovery rates. It is reasonable to assume that non-opinionated individuals are more likely to form an opinion if there are more opinionated individuals. Therefore, we assume that the infection rate of susceptible individuals is an affine function of the number of infected individuals,

$$
\alpha_{s i}(\mathbf{x})=\left(\alpha_{s i}^{0}+\alpha_{s i}^{1} x_{i}\right) x_{s} .
$$

Moreover, the effects of other individuals on shifting to neutral (recovered) are neglected. Therefore the rate at which non-opinionated and opinionated individuals shift to neutral are constant, which implies,

$$
\alpha_{i r}(\mathbf{x})=\alpha_{i r}^{0} x_{i}, \quad \alpha_{s r}(\mathbf{x})=\alpha_{s r}^{0} x_{s}
$$

Finally, having many individuals in the community, is likely to attract new opinionated and non-opinionated individuals. Hence, we assume that the arrival rates of the susceptible and infected individuals are affine functions of the total number of individuals,

$$
\lambda_{i}(\mathbf{x})=\lambda_{i}^{0}+\lambda_{i}^{1}\|\mathbf{x}\|_{1}, \quad \lambda_{s}(\mathbf{x})=\lambda_{s}^{0}+\lambda_{s}^{1}\|\mathbf{x}\|_{1}
$$

whereas neutral individuals arrive at a constant rate,

$$
\lambda_{r}(\mathbf{x})=\lambda_{r}^{0} .
$$

Note that the assumptions above are a generalisation of Kermack and McKendrick's SIR model. In Kermack and McKendrick's setting, the population is fixed $\left(\lambda_{i}^{0}=\lambda_{i}^{1}=\lambda_{s}^{0}=\lambda_{s}^{1}=\lambda_{r}^{0}=\mu=0\right)$, the infection rate is proportional to the number of infected individuals $\left(\alpha_{s i}^{0}=0\right)$, and individuals do not recover without being infected $\left(\alpha_{s r}^{0}=0\right)$.

\section{Maclaurin-series expansions}

While the system of equations (1) is easily solved when the maximum number of individuals is limited, the state space size already explodes for relatively small $L$ and a direct solution is computationally infeasible. Indeed, numerical computation of the steady-state vector has an asymptotic time complexity of $O\left(M^{3}\right)$, where $M=\left(\begin{array}{c}L+3 \\ 3\end{array}\right) \sim L^{3} / 6$ is the size of the state space. We introduce the numerical Maclaurin-series approach in generic terms in the subsection below and survey related work in subsection 3.2. We then tailor the method to the Markov chain at hand in subsection 3.3 and derive performance measures in subsection 3.4 . 


\subsection{Methodology}

Notice that the generator matrix of the Markov chain at hand can be decomposed as follows,

$$
Q_{\mu}=Q^{(0)}+\mu Q^{(1)},
$$

with neither $Q^{(0)}$ nor $Q^{(1)}$ depending on $\mu$. Moreover, $Q^{(0)}$ is still a proper generator matrix, it is the generator matrix of the Markov process when there are no departures. As the size of the state space does not allow for a direct calculation of $\pi$ as the normalised solution of,

$$
\pi Q_{\mu}=0,
$$

we introduce the Maclaurin-series expansion of the steady state vector in $\mu$,

$$
\pi=\sum_{n=0}^{\infty} \mu^{n} \pi_{n} .
$$

In order for such an expansion to make sense, the vector $\pi$ is required to be analytic in a neighbourhood of $\mu=0$. For finite state spaces (in contrast to infinite ones, see e.g. $[3,18]$ ), this is fairly easy to establish. Finding the steady-state distribution is in this case essentially a finite-dimensional eigenproblem. If a matrix depends analytically on a parameter, then the corresponding eigenvalues and eigenvectors are also analytic in case of null-space perturbation [1]. Another possible path towards proving analyticity is via $V$-uniform ergodicity of the unperturbed Markov process with generator $Q^{(0)}$ (see a.o [3]), which is equivalent to the existence of a spectral gap (the distance between eigenvalue 0 of the generator matrix $Q^{(0)}$ and the eigenvalue that is its nearest neighbour). For finite Markov chains, there is a spectral gap as long as there is only one recurrent class for $\mu=0$. Hence, provided that $Q^{(0)}$ is a generator matrix with one recurrent class (this condition is also denoted as 'regular perturbation', as opposed to 'singular perturbation'), the expansion makes sense and $\pi_{0}$ is the normalised solution of the equation,

$$
\pi_{0} Q^{(0)}=0 .
$$

Every subsequent term $\pi_{n}$ can be found by identifying equal powers of $\mu$ in the equation,

$$
\sum_{n=0}^{\infty} \mu^{n} \pi_{n}\left(Q^{(0)}+\mu Q^{(1)}\right)=0,
$$

which leads to the following equation for $\pi_{n+1}$,

$$
\pi_{n+1} Q^{(0)}=-\pi_{n} Q^{(1)} .
$$

The normalisation condition of $\pi$ implies that the elements of $\pi_{n}$ sum to 0 for each $n>0$. This fact and the former equation allows for recursively solving the terms in the expansion.

As we now have to solve a linear system of equations for each term $\pi_{n}$ in the expansion, plus an additional vector-matrix product, it appears that we have not gained very much. However, if we impose the 
extra condition that $Q^{(0)}$ is triangular for some ordering of the state space (either upper or lower triangular), then the resulting linear systems of equations can be solved by backward substitutions, with considerably reduced computational complexity. As a worst case, its computation time is $O\left(M^{2}\right)$. However, the number of transitions from a state is typically much smaller than the state space, such that the computation time typically is $O(M)$.

\subsection{Related work}

Prior to applying the series expansion method to the Markov model at hand, we survey work on series expansions of stochastic models. For an overview, we refer the reader to [6], [27] and the recent book [2]. The first work on series expansions of stochastic models seems to be by Schweitzer in 1968 [35]. Ever since, it has been applied in many forms and flavours, and is known under various names such as perturbations, light-traffic expansions, Taylor-series expansions and so on. This technique is in principle not confined to the Markov framework (see e.g. [7], which utilises Palm theory), although many interesting examples indeed fall within this framework.

There are roughly three methods to establish series expansions of stochastic models. The first makes use of the direct derivation sketched above and forms the basis of the computational method that we propose in this paper and will evaluate in the next subsection. The second makes use of sample-path arguments. Consider the case that $\mu$ denotes a particular event rate. For example, for light-traffic approximations, $\mu$ denotes the arrival rate; in the worked-out example of section 3, the parameter denotes the service rate, and hence constitutes a 'low-service rate' approximation. An important result for this strand of research is what we can call the $n$ events rule, which states that for an $n$th order expansion, only sample paths with $n$ or fewer of such events must be considered. This can be intuited from the non-rigorous reasoning that a sample path containing $n$ such events has a probability of order $\mu^{n}$. However due to the fact that the number of sample paths is uncountable and thus the probability of every individual path is zero, making this rigorous is non-trivial. For series expansions revolving around a Poisson process with a small rate, to which the examples in this work essentially belong, this was made rigorous by Reiman and Simon [32]. Important work extending this to a Palm calculus context was presented in [7].

The third approach to series expansions is via the following updating formula, which has been established in general Markov settings, see eg. [18]:

$$
\pi=\pi_{0} \sum_{k=0}^{\infty}\left[\mu Q^{(1)} D\right]^{k}
$$

where $D$ denotes the deviation matrix of $Q^{(0)}$. In this case, a successful application revolves around finding this deviation matrix $D$, defined as follows:

$$
D=\int_{0}^{\infty}\left(\left[P_{0}(t)\right]_{i j}-\Pi_{0}\right) d t
$$


with $P_{0}(t)$ the Markov semigroup of the continuous-time Markov chain and $\Pi_{0}=\lim _{t \rightarrow \infty} P_{0}(t)$.

As the matrix $D$ is closely related to Poisson's equation for Markov chains, this technique is sometimes also denoted as such [30]. Note that the matrix $D$ pertains to the unperturbed Markov chain, so that in this updating formula we see another justification for the $n$ events rule. Indeed, as the events are in fact nothing else than the transitions recorded in $Q^{(1)}$, transitions which do not occur in $Q^{(0)}$ and hence nor in $D$, it follows that in the vector $\pi_{n}=\pi_{0}\left(Q^{(1)} D\right)^{n}$, only those states that can be reached with $n$ events (or less) can be non-zero. To the best of our knowledge, a rigourous identification of the sample-path method and the updating formula has not yet been attempted.

\subsection{Application}

In view of the method described above, let $\pi_{n}(\mathbf{x})$ be the $n$th component of the expansion,

$$
\pi(\mathbf{x})=\sum_{n=0}^{\infty} \pi_{n}(\mathbf{x}) \mu^{n}
$$

for $\mathbf{x} \in \mathcal{L}$. Substituting the former expression in the balance equations (1), we get

$$
\begin{aligned}
& \sum_{n=0}^{\infty} \pi_{n}(\mathbf{x}) \mu^{n}\left(\|\mathbf{x}\|_{1} \mu+\lambda(\mathbf{x})+\alpha_{s i}(\mathbf{x})+\alpha_{i r}(\mathbf{x})+\alpha_{s r}(\mathbf{x})\right) \\
& =\mathbb{1}_{\left\{\|\mathbf{x}\|_{1}<L\right\}}\left(\sum_{n=0}^{\infty} \pi_{n}\left(x_{r}+1, x_{i}, x_{s}\right) \mu^{n+1}\left(x_{r}+1\right)+\sum_{n=0}^{\infty} \pi_{n}\left(x_{r}, x_{i}+1, x_{s}\right) \mu^{n+1}\left(x_{i}+1\right)\right. \\
& \left.\quad+\sum_{n=0}^{\infty} \pi_{n}\left(x_{r}, x_{i}, x_{s}+1\right) \mu^{n+1}\left(x_{s}+1\right)\right)+\sum_{n=0}^{\infty} \pi_{n}\left(x_{r}-1, x_{i}, x_{s}\right) \lambda_{r}\left(x_{r}-1, x_{i}, x_{s}\right) \mu^{n} \\
& \quad+\sum_{n=0}^{\infty} \pi_{n}\left(x_{r}, x_{i}-1, x_{s}\right) \lambda_{i}\left(x_{r}, x_{i}-1, x_{s}\right) \mu^{n}+\sum_{n=0}^{\infty} \pi_{n}\left(x_{r}, x_{i}, x_{s}-1\right) \lambda_{s}\left(x_{r}, x_{i}, x_{s}-1\right) \mu^{n} \\
& +\sum_{n=0}^{\infty} \pi_{n}\left(x_{r}, x_{i}-1, x_{s}+1\right) \alpha_{s i}\left(x_{r}, x_{i}-1, x_{s}+1\right) \mu^{n}+\sum_{n=0}^{\infty} \pi_{n}\left(x_{r}-1, x_{i}+1, x_{s}\right) \alpha_{i r}\left(x_{r}-1, x_{i}+1, x_{s}\right) \mu^{n} \\
& +\sum_{n=0}^{\infty} \pi_{n}\left(x_{r}-1, x_{i}, x_{s}+1\right) \alpha_{s r}\left(x_{r}-1, x_{i}, x_{s}+1\right) \mu^{n}
\end{aligned}
$$

For $\mathbf{x} \in \mathcal{L}^{*}=\mathcal{L} \backslash\{(L, 0,0)\}$, comparison of the terms in $\mu^{0}$ on both sides of the former equation yields,

$$
\begin{aligned}
& \pi_{0}(\mathbf{x})(\lambda(\mathbf{x})\left.+\alpha_{s i}(\mathbf{x})+\alpha_{i r}(\mathbf{x})+\alpha_{s r}(\mathbf{x})\right) \\
& \quad=\sum_{n=0}^{\infty} \pi_{0}\left(x_{r}-1, x_{i}, x_{s}\right) \lambda_{r}\left(x_{r}-1, x_{i}, x_{s}\right)+\sum_{n=0}^{\infty} \pi_{0}\left(x_{r}, x_{i}-1, x_{s}\right) \lambda_{i}\left(x_{r}, x_{i}-1, x_{s}\right) \\
& \quad+\sum_{n=0}^{\infty} \pi_{0}\left(x_{r}, x_{i}, x_{s}-1\right) \lambda_{s}\left(x_{r}, x_{i}, x_{s}-1\right)+\sum_{n=0}^{\infty} \pi_{0}\left(x_{r}, x_{i}-1, x_{s}+1\right) \alpha_{s i}\left(x_{r}, x_{i}-1, x_{s}+1\right) \\
&+\sum_{n=0}^{\infty} \pi_{0}\left(x_{r}-1, x_{i}+1, x_{s}\right) \alpha_{i r}\left(x_{r}-1, x_{i}+1, x_{s}\right)+\sum_{n=0}^{\infty} \pi_{0}\left(x_{r}-1, x_{i}, x_{s}+1\right) \alpha_{s r}\left(x_{r}-1, x_{i}, x_{s}+1\right) .
\end{aligned}
$$


Evaluating this expression in lexicographical order shows,

$$
\pi_{0}(\mathbf{x})=0
$$

for $\mathbf{x} \in \mathcal{L} \backslash\{(L, 0,0)\}$. By the normalisation condition of $\pi_{0}$ we further get $\pi_{0}(L, 0,0)=1$. This result is not unexpected. In the absence of departures, the population size reaches its boundary and no new arrivals are possible. Moreover, every individual in the population will recover after some time, such that there are but recovered individuals.

Comparison of the terms in $\mu^{n}$ for $n>0$ in equation (4) gives,

$$
\begin{aligned}
& \quad \pi_{n}(\mathbf{x})=\frac{1}{\Delta(\mathbf{x})}\left(\mathbb { 1 } _ { \{ \| \mathbf { x } | _ { 1 } < L \} } \left(\pi_{n-1}\left(x_{r}+1, x_{i}, x_{s}\right)\left(x_{r}+1\right)+\pi_{n-1}\left(x_{r}, x_{i}+1, x_{s}\right)\left(x_{i}+1\right)\right.\right. \\
& \left.+\pi_{n-1}\left(x_{r}, x_{i}, x_{s}+1\right)\left(x_{s}+1\right)\right)+\pi_{n}\left(x_{r}-1, x_{i}, x_{s}\right) \lambda_{r}\left(x_{r}-1, x_{i}, x_{s}\right)+\pi_{n}\left(x_{r}, x_{i}-1, x_{s}\right) \lambda_{i}\left(x_{r}, x_{i}-1, x_{s}\right) \\
& \quad+\pi_{n}\left(x_{r}, x_{i}, x_{s}-1\right) \lambda_{s}\left(x_{r}, x_{i}, x_{s}-1\right)+\pi_{n}\left(x_{r}, x_{i}-1, x_{s}+1\right) \alpha_{s i}\left(x_{r}, x_{i}-1, x_{s}+1\right) \\
& \left.+\pi_{n}\left(x_{r}-1, x_{i}+1, x_{s}\right) \alpha_{i r}\left(x_{r}-1, x_{i}+1, x_{s}\right)+\pi_{n}\left(x_{r}-1, x_{i}, x_{s}+1\right) \alpha_{s r}\left(x_{r}-1, x_{i}, x_{s}+1\right)-\pi_{n-1}(\mathbf{x})\|\mathbf{x}\|_{1}\right)
\end{aligned}
$$

for $\mathbf{x} \in \mathcal{L} \backslash\{(L, 0,0)\}$ with,

$$
\Delta(\mathbf{x})=\left(\lambda(\mathbf{x})+\alpha_{s i}(\mathbf{x})+\alpha_{i r}(\mathbf{x})+\alpha_{s r}(\mathbf{x})\right)
$$

As detailed in [13], we can use the above equation to compute new terms very efficiently, by iterating over the state space in lexicographic order, as on the RHS only entries of either order $n-1$ or lexicographically smaller entries of order $n$ are present. Moreover, the assumptions on the arrival process assure that $\Delta(\mathbf{x})>0$ for all $\mathbf{x} \in \mathcal{L} \backslash\{(L, 0,0)\}$. As for the 0th order term, the normalisation condition is used to find the $n$th order expansion of $\pi(L, 0,0)$,

$$
\pi_{n}(L, 0,0)=-\sum_{\mathbf{x} \in \mathcal{L} \backslash\{(L, 0,0)\}} \pi_{n}(\mathbf{x})
$$

\subsection{Performance measures}

Once the series expansion of the steady-state distribution has been obtained, the expansion of various performance measures directly follows. Let $\mathbf{X} \sim \pi$, then for a performance measure $J=\mathrm{E}[f(\mathbf{X})]$, we have

$$
J=\sum_{\mathbf{x} \in \mathcal{L}} f(\mathbf{x}) \pi(\mathbf{x})=\sum_{\mathbf{x} \in \mathcal{L}} f(\mathbf{x}) \sum_{n=0}^{\infty} \pi_{n}(\mathbf{x}) \mu^{n}=\sum_{n=0}^{\infty} \sum_{\mathbf{x} \in \mathcal{L}} f(\mathbf{x}) \pi_{n}(\mathbf{x}) \mu^{n}=\sum_{n=0}^{\infty} J_{n} \mu^{n},
$$

with

$$
J_{n}=\sum_{\mathbf{x} \in \mathcal{L}} f(\mathbf{x}) \pi_{n}(\mathbf{x}) .
$$

The interchange of the summations is justified by the finiteness of $\mathcal{L}$ and the convergence of $\sum_{n} \pi_{n}(\mathbf{x}) \mu^{n}$ for all $\mathbf{x} \in \mathcal{L}$. As such, any term $J_{n}$ in the expansion of a performance measure $J$ can be calculated from the corresponding vector $\pi_{n}$ of the expansion of the steady-state vector $\pi$. Performance measures of interest include amongst others the $j$ th order moment of the number of individuals of type $k \in \mathcal{K}\left(f(\mathbf{x})=x_{k}{ }^{j}\right)$. 


\section{Fluid limit}

In this section, we develop a fluid limit for the model described in this contribution, relying on the monograph of Ethier and Kurtz [15], and on the survey article by Darling and Norris [10]. We study this Markov model via its generator $Q$,

$$
Q f(\mathbf{x}):=\lim _{t \rightarrow 0} \frac{1}{t}(\mathrm{E}[f(\mathbf{X}(t)) \mid X(0)=\mathbf{x}]-f(\mathbf{x})),
$$

for any bounded and measurable function $f: \mathcal{L} \rightarrow \mathbb{R}$. It is straightforward to deduce from the informal description of section 2 that

$$
\begin{aligned}
Q f(\mathbf{x}) & =\sum_{k \in \mathcal{K}} \lambda_{k}(\mathbf{x})\left[f\left(\mathbf{x}+\mathbf{e}_{k}\right)-f(\mathbf{x})\right]+\sum_{k \in \mathcal{K}} \mu x_{k}\left[f\left(\mathbf{x}-\mathbf{e}_{k}\right)-f(\mathbf{x})\right] \\
& +\sum_{(j, k) \in \mathcal{K}^{*}} \alpha_{j k}(\mathbf{x})\left[f\left(\mathbf{x}-\mathbf{e}_{j}+\mathbf{e}_{k}\right)-f(\mathbf{x})\right],
\end{aligned}
$$

where $\mathbf{e}_{r}:=[1,0,0], \mathbf{e}_{i}:=[0,1,0], \mathbf{e}_{s}:=[0,0,1]$ and $\mathcal{K}^{*}:=\{(s, i),(i, r),(s, r)\}$.

\subsection{Convergence for the generic epidemic process}

Let $\left\{X_{r}^{\varepsilon}(t), X_{i}^{\varepsilon}(t), X_{s}^{\varepsilon}(t)\right\}$ denote the continuous-time Markov process indexed by the scaling parameter $\varepsilon$, which affects the system in the following way: step sizes are scaled by $\varepsilon$, whereas the transition rates are scaled by $\varepsilon^{-1}$. We assume that the Markov process takes values in a compact set $U \subset \mathbb{R}^{3}$, where $U:=\left\{\mathbf{x} \in \mathbb{R}^{3}: \mathbf{x} \geq \mathbf{0},\|\mathbf{x}\|_{1} \leq L\right\}$.

In particular, the generator of the scaled process is as follows,

$$
\begin{aligned}
Q_{\varepsilon} f(\mathbf{x}) & =\sum_{k \in \mathcal{K}} \varepsilon^{-1} \bar{\lambda}_{k}(\mathbf{x})\left[f\left(\mathbf{x}+\varepsilon \mathbf{e}_{k}\right)-f(\mathbf{x})\right]+\sum_{k \in \mathcal{K}} \varepsilon^{-1} \mu x_{k}\left[f\left(\mathbf{x}-\varepsilon \mathbf{e}_{k}\right)-f(\mathbf{x})\right] \\
& +\sum_{(j, k) \in \mathcal{K}^{*}} \varepsilon^{-1} \bar{\alpha}_{j k}(\mathbf{x})\left[f\left(\mathbf{x}-\varepsilon \mathbf{e}_{j}+\varepsilon \mathbf{e}_{k}\right)-f(\mathbf{x})\right]
\end{aligned}
$$

for suitable functions $\bar{\lambda}_{k}(\cdot)$ and $k \in \mathcal{K}, \bar{\alpha}_{j k}(\cdot),(j, k) \in \mathcal{K}^{*}$, which we require to be Lipschitz continuous on $U$. The functions $\bar{\lambda}_{k}(\cdot)$ extend the domain of the functions $\lambda_{k}(\cdot)$ to $\mathcal{L}$. Note that Lipschitz continuity means in particular that the arrival rates $\bar{\lambda}_{k}(\cdot)$ must not go discontinuously to zero at the border $\partial U$ of $U$, and therefore we smoothen these functions in a certain manner. In terms of numerical results, the exact manner with which these are smoothened is not important, as we have found that the fluid limit gives the best results when the system steers clear from the boundary as $\varepsilon \rightarrow 0$, that is

$$
\operatorname{Pr}\left[d\left(\mathbf{X}^{\varepsilon}(t), \partial U\right)>\delta\right] \rightarrow 0,
$$

where $d(\cdot, \cdot)$ denotes the Euclidean distance. 
Let us introduce the transition rates $q_{\varepsilon}\left(\mathbf{x}, \mathbf{x}^{\prime}\right)$ corresponding to $Q_{\varepsilon}$ in the obvious manner. By 'Tayloring', we find that

$$
\begin{aligned}
Q_{\varepsilon} f(\mathbf{x}) & \rightarrow \sum_{k \in \mathcal{K}} \bar{\lambda}_{k}(\mathbf{x}) \partial_{x_{k}} f(\mathbf{x})-\sum_{k \in \mathcal{K}} \mu x_{k} \partial_{x_{k}} f(\mathbf{x}) \\
& +\sum_{(j, k) \in \mathcal{K}^{*}} \bar{\alpha}_{j k}(\mathbf{x})\left[-\partial_{x_{j}}+\partial_{x_{k}}\right] f(\mathbf{x})+O\left(\varepsilon^{2}\right) .
\end{aligned}
$$

Note that the limit is exact when $f$ is a linear function, as the second order derivatives of $f$, which feature in the $O\left(\varepsilon^{2}\right)$ term, are evidently all zero. It is well-known that a generator of this type has a deterministic solution that can be formulated in terms of the following system of (non-linear) differential equations:

$$
\begin{aligned}
& \dot{x}_{s}(t)=\lambda_{s}(\mathbf{x}(t))-\bar{\alpha}_{s i}(\mathbf{x}(t))-\bar{\alpha}_{s r}(\mathbf{x}(t))-\mu x_{s}(t) \\
& \dot{x}_{i}(t)=\lambda_{i}(\mathbf{x}(t))+\bar{\alpha}_{s i}(\mathbf{x}(t))-\bar{\alpha}_{i r}(\mathbf{x}(t))-\mu x_{i}(t) \\
& \dot{x}_{r}(t)=\lambda_{r}(\mathbf{x}(t))+\bar{\alpha}_{i r}(\mathbf{x}(t))+\bar{\alpha}_{s r}(\mathbf{x}(t))-\mu x_{r}(t)
\end{aligned}
$$

which has in general no closed-form solution but can be solved efficiently with a suitable numerical procedure for differential equations. Let us denote this system of equations in shorthand as $\dot{\mathbf{x}}=\mathbf{b}(\mathbf{x})$, for a suitably defined vector field $\mathbf{b}(\cdot)$ on $U$, with Lipschitz constant $K$.

We show an error bound for the fluid limit using Theorem 4.2 from [10]. Consider the following events:

$$
\begin{gathered}
\Omega_{0}:=\left\{\left\|\mathbf{X}^{\varepsilon}(0)-\mathbf{x}(0)\right\| \leq \delta\right\}, \\
\Omega_{1}:=\left\{\int_{0}^{t_{0}}\left\|\beta\left(\mathbf{X}^{\varepsilon}(t)\right)-\mathbf{b}(\mathbf{x}(t))\right\| d t \leq \delta\right\}, \\
\Omega_{2}:=\left\{\int_{0}^{t_{0}} \gamma\left(\mathbf{X}^{\varepsilon}(t)\right) d t \leq A(\varepsilon) t_{0}\right\} .
\end{gathered}
$$

where

$$
\beta(\mathbf{x})=\sum_{\mathbf{x}^{\prime}} q\left(\mathbf{x}, \mathbf{x}^{\prime}\right)\left(\mathbf{x}^{\prime}-\mathbf{x}\right),
$$

and

$$
\gamma(\mathbf{x})=\sum_{\mathbf{x}^{\prime}} q\left(\mathbf{x}, \mathbf{x}^{\prime}\right)\left\|\mathbf{x}^{\prime}-\mathbf{x}\right\|^{2}
$$

Here $\|\cdot\|$ is the Euclidean norm.

We have per Theorem 4.2 that

$$
\mathbb{P}\left(\sup _{t \leq t_{0}}\left\|\mathbf{X}^{\varepsilon}(t)-\mathbf{x}\right\|>\kappa(\varepsilon)\right) \leq 4 A(\varepsilon) t_{0} / \delta^{2}+\mathbb{P}\left(\Omega_{0}^{c} \cup \Omega_{1}^{c} \cup \Omega_{2}^{c}\right) .
$$

where $\delta=\kappa(\varepsilon) e^{-K t_{0}} / 3$. 
A simple calculation shows that $\mathbb{P}\left(\Omega_{1}^{c}\right)=0$ and also $\mathbb{P}\left(\Omega_{2}^{c}\right)=0$ if we choose

$$
\varepsilon^{-1} A(\varepsilon)=\sum_{k \in \mathcal{K}} \sup _{\mathbf{x}} \bar{\lambda}_{k}(\mathbf{x})+\mu L+\sqrt{2} \sum_{(j, k) \in \mathcal{K}^{*}} \sup _{\mathbf{x}} \bar{\alpha}_{j k}(\mathbf{x}) .
$$

If we choose $\kappa(\varepsilon)$ such that $\kappa(\varepsilon)^{2} / \varepsilon \rightarrow 0$, and $\mathbf{X}^{\varepsilon}(0) \rightarrow \mathbf{x}(0)$ then we have indeed convergence to the fluid limit.

\subsection{Equilibrium points for the opinion spreading model}

In this section, we calculate the equilibrium points for the opinion spreading model introduced in subsection 2.2 by solving $\mathbf{b}(\mathbf{x})=\mathbf{0}$. We assume all rates are positive: $\alpha_{s i}^{0}>0, \alpha_{s i}^{1}>0, \alpha_{i r}^{0}>0, \alpha_{s r}^{0}>0, \lambda_{s}^{0}>0$, $\lambda_{s}^{1}>0, \lambda_{i}^{0}>0, \lambda_{i}^{1}>0$ and $\lambda_{r}^{0}>0$. In view of the assumptions on the rates introduced in section 2.2 , we find the following set of equations for the equilibrium points of the opinion spreading model,

$$
\begin{aligned}
& 0=\left(\lambda_{s}^{0}+\lambda_{s}^{1}\|\mathbf{x}\|_{1}\right) \mathbb{1}_{\left\{\|\mathbf{x}\|_{1}<L\right\}}-\left(\alpha_{s i}^{0}+\alpha_{s i}^{1} x_{i}\right) x_{s}-\alpha_{s r}^{0} x_{s}-\mu x_{s} \\
& 0=\left(\lambda_{i}^{0}+\lambda_{i}^{1}\|\mathbf{x}\|_{1}\right) \mathbb{1}_{\left\{\|\mathbf{x}\|_{1}<L\right\}}+\left(\alpha_{s i}^{0}+\alpha_{s i}^{1} x_{i}\right) x_{s}-\alpha_{i r}^{0} x_{i}-\mu x_{i} \\
& 0=\lambda_{r}^{0} \mathbb{1}_{\left\{\|\mathbf{x}\|_{1}<L\right\}}+\alpha_{i r}^{0} x_{i}+\alpha_{s r}^{0} x_{s}-\mu x_{r}
\end{aligned}
$$

where $\|\mathbf{x}\|_{1}=x_{r}+x_{i}+x_{s}$.

First assume $\|\mathbf{x}\|_{1}=L$. Then $x_{s}=0$ implies $x_{i}=x_{r}=0$ by equations (14) and (15) and hence $\|\mathbf{x}\|_{1} \neq L$. In addition $x_{s}>0$ implies $x_{r}<0$ by equation (14). We conclude that there is no non-negative equilibrium point for $\|\mathbf{x}\|_{1}=L$.

Now assume $\|\mathbf{x}\|_{1}<L$. By subtracting (13) from (14), we can eliminate the quadratic part in $\alpha_{s i}(\mathbf{x})$. Then, we find solutions for $x_{i}$ and $x_{r}$ by solving this new linear equation together with equation (15). These solutions are substituted in equation (13) such that we get the following quadratic equation in $x_{s}$ :

$$
\begin{array}{r}
x_{s}^{2}+\left(\frac{\lambda_{r}^{0}}{\mu+\alpha_{s r}^{0}}-\frac{\mu\left(\lambda_{i}^{0}+\lambda_{r}^{0}+\lambda_{s}^{0}\right)}{\left(\mu-\lambda_{i}^{1}-\lambda_{s}^{1}\right)\left(\mu+\alpha_{s r}^{0}\right)}-\frac{\alpha_{s i}^{0}\left(\mu+\alpha_{i r}^{0}\right)}{\left(\mu+\alpha_{s r}^{0}\right) \alpha_{s i}^{1}}-\frac{\mu+\alpha_{i r}^{0}}{\alpha_{s i}^{1}}\right) x_{s} \\
+\frac{\left(\mu \lambda_{s}^{0}+\lambda_{i}^{0} \lambda_{s}^{1}-\lambda_{i}^{1} \lambda_{s}^{0}+\lambda_{r}^{0} \lambda_{s}^{1}\right)\left(\mu+\alpha_{i r}^{0}\right)}{\alpha_{s i}^{1}\left(\mu-\lambda_{i}^{1}-\lambda_{s}^{1}\right)\left(\mu+\alpha_{s r}^{0}\right)}=0 .
\end{array}
$$

For both solutions $x_{s}$ of the quadratic equation above, we find $x_{i}$ in terms of $x_{s}$,

$$
x_{i}=\frac{\left(\lambda_{i}^{1} \mu+\alpha_{s i}^{0} \lambda_{i}^{1}+\alpha_{s i}^{0} \lambda_{s}^{1}+\lambda_{i}^{1} \alpha_{s r}^{0}\right) x_{s}+\lambda_{i}^{0} \lambda_{s}^{1}-\lambda_{i}^{1} \lambda_{s}^{0}}{\lambda_{s}^{1}\left(\mu+\alpha_{i r}^{0}\right)-\alpha_{s i}^{1}\left(\lambda_{i}^{1}+\lambda_{s}^{1}\right) x_{s}},
$$

and $x_{r}$ in terms of $x_{i}$ and $x_{s}$,

$$
x_{r}=\frac{\alpha_{i r}^{0} x_{i}+\alpha_{s r}^{0} x_{s}+\lambda_{r}^{0}}{\mu} .
$$


To examine the stability of the equilibrium point, we investigate the Jacobian matrix at each of the equilibrium points from the quadratic equation. We have

$$
\mathcal{J}=\left[\begin{array}{lll}
\frac{\mathrm{d} F_{s}}{\mathrm{~d} x_{s}} & \frac{\mathrm{d} F_{i}}{\mathrm{~d} x_{s}} & \frac{\mathrm{d} F_{r}}{\mathrm{~d} x_{s}} \\
\frac{\mathrm{d} F_{s}}{\mathrm{~d} x_{i}} & \frac{\mathrm{d} F_{i}}{\mathrm{~d} x_{i}} & \frac{\mathrm{d} F_{r}}{\mathrm{~d} x_{i}} \\
\frac{\mathrm{d} F_{s}}{\mathrm{~d} x_{r}} & \frac{\mathrm{d} F_{i}}{\mathrm{~d} x_{r}} & \frac{\mathrm{d} F_{r}}{\mathrm{~d} x_{r}}
\end{array}\right]
$$

where $F_{s}, F_{i}$ and $F_{r}$ are respectively equal to the right hand sides of equations (13), (14) and (15). We get

$$
\mathcal{J}=\left[\begin{array}{ccc}
\lambda_{s}^{1}-\alpha_{s i}^{0}-\alpha_{s i}^{1} x_{i}-\mu & \lambda_{i}^{1}+\alpha_{s i}^{0}+\alpha_{s i}^{1} x_{i} & \alpha_{s r}^{0} \\
\lambda_{s}^{1}-\alpha_{s i}^{1} x_{s} & \lambda_{i}^{1}+\alpha_{s i}^{1} x_{s}-\alpha_{i r}^{0}-\mu & \alpha_{i r}^{0} \\
\lambda_{s}^{1} & \lambda_{i}^{1} & -\mu
\end{array}\right] .
$$

Note that if all eigenvalues have a negative real part, the equilibrium point is stable, otherwise the equilibrium point is unstable. Numerical results will be given in the next section.

\section{Numerical results}

To illustrate our numerical approach, we now assess the accuracy of the series expansion technique and the fluid limit by means of several numerical examples.

\subsection{Constant infection and arrival rates}

First, consider the first example as described in subsection 2.1. Recall, that for this particular example, the solution can be calculated explicitly. We here compare the accuracy of the series expansion, with the exact result. To this end, figures 2(a) and 2(b) depict the mean number of infected and susceptible individuals, respectively, versus the arrival rate of infected individuals $\lambda_{i}$. The maximum population size is $L=50$ and we further assume $\lambda_{r}=\lambda_{s}=1$ and $\alpha_{i r}=\alpha_{s i}=\alpha_{s r}=1$. Moreover, the departure rate is set to $\mu=0.05$. The exact result is compared with the approximation by an $N$ th order expansion in $\mu$ for $N=2$, $N=4$ and $N=8$. For $N=8$, we observe that the approximation is accurate, apart from a slight deviation for small $\lambda_{i}$. In contrast, for $N=2$ and $N=4$, the results of the series expansions are clearly not accurate for the considered parameter settings.

To establish the regions in which the results of the series expansion are accurate enough, we propose a simple heuristic which compares the $N$ th and the $2 N$ th order expansions. Let $f_{N}(\mu)$ be the $N$ th order expansion in $\mu$, we then accept our $N$ th order approximation provided if

$$
\frac{\left|f_{2 N}(\mu)-f_{N}(\mu)\right|}{f_{2 N}(\mu)}<\epsilon,
$$

or equivalently,

$$
1-\epsilon<\frac{f_{N}(\mu)}{f_{2 N}(\mu)}<1+\epsilon .
$$




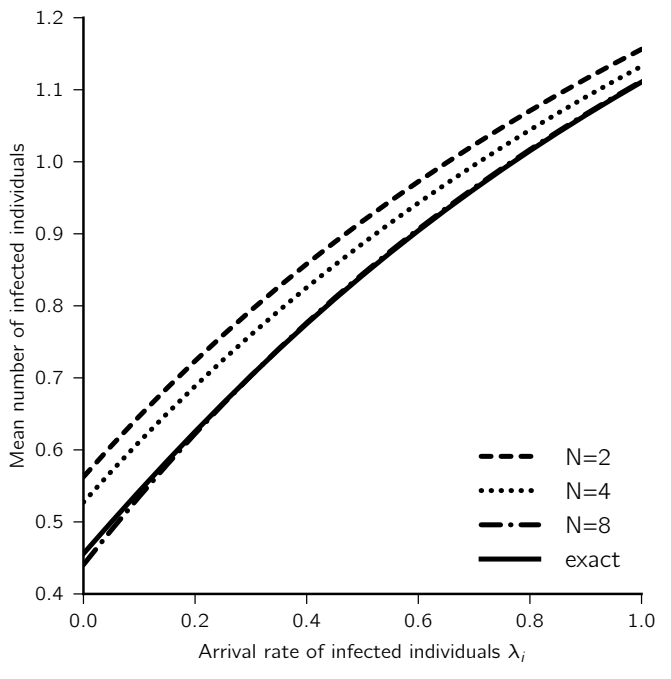

(a)

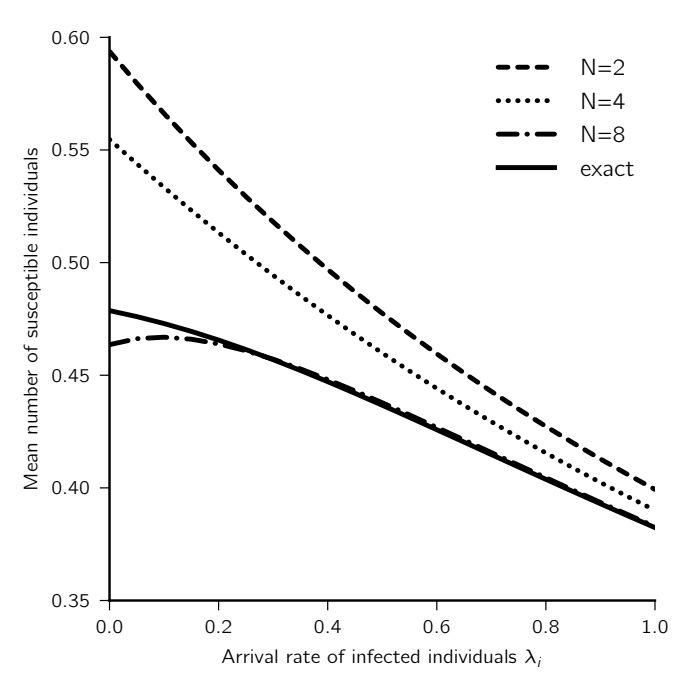

(b)

Figure 2: Mean number of infected (a) and susceptible (b) individuals.

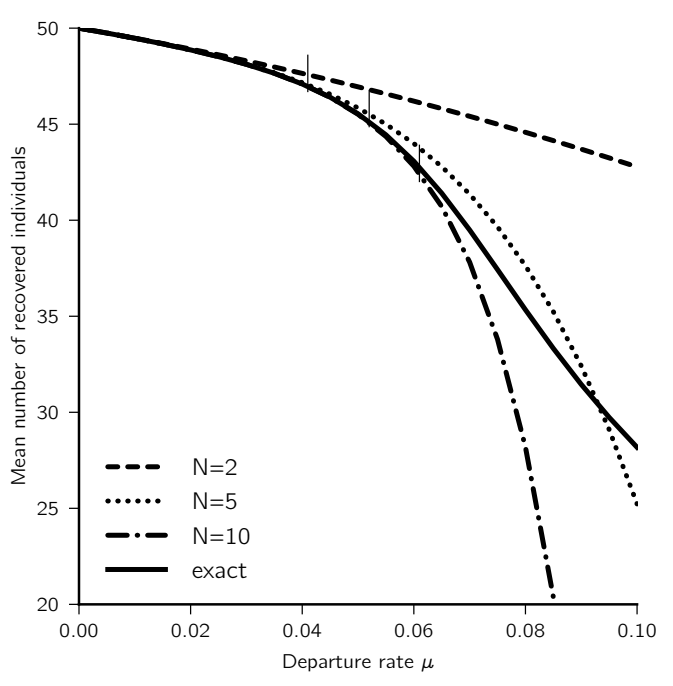

Figure 3: Mean number of recovered individuals. 
Let $\Omega_{N}$ denote the region where these inequalities hold. In Figure 3 which shows the mean number of recovered individuals versus the departure rate $\mu$, we divide the region where these inequalities hold and do not hold for $N=\{2,5,10\}$ with $\epsilon=0.01$ by means of a line. The same parameter settings as in Figures $2(\mathrm{a})$ and $2(\mathrm{~b})$ are considered and the arrival rate of infected individuals is assumed to be equal to 1 . As can be observed, the regions for which the inequality of the heuristic hold go up to $\mu=0.041$ for $N=2$, up to $\mu=0.052$ for $N=5$ and up to $\mu=0.061$ for $N=10$. Comparing the results of the approximation method with the exact result, we observe that the performance assessment is accurate in the heuristically determined region.

\subsection{Opinion spreading model}

We now consider the opinion spreading model as described in section 2.2. In this case, the exact solution cannot be calculated and we rely on simulation to assess the accuracy of our results. Figures 4(a), 4(b) and 4(c) depict respectively the mean number of recovered individuals, the mean number of infected individuals and the mean number of susceptible individuals versus the lifetime rate $\mu$ varying from 0 to 0.6 . Moreover, the maximum population size equals $L=20$, the arrival rates $\lambda_{k}^{0}$ where $k=\{r, i, s\}$ and $\lambda_{i}^{1}$ where $i=\{i, s\}$ are respectively equal to 3 and 0.1 and the rates at which an individual changes of type, $\alpha_{s i}^{0}, \alpha_{s i}^{1}, \alpha_{s r}^{0}$ and $\alpha_{i r}^{0}$, are equal to 3 . Series expansions of various orders $N$ are depicted as indicated $(N=1,5,10)$, as well as simulation results. Here and in the following figures, the $99 \%$ confidence interval of the simulation results was determined by the batch means method. The size of the confidence however does not exceed $1 \%$ of the value and is therefore omitted on the plot. As expected, the mean number of recovered individuals decreases and the mean number of infected and susceptible individuals increase as the departure rate increases. Moreover, for $\mu=0$, the population consists only of recovered individuals as their lifetime is infinite such that all individuals recover eventually. As the figures show, the approximation for $N=5$ is already accurate for the mean number of recovered, infected and susceptible individuals.

We also consider the fluid approximation of the opinion spreading model. In Figure 5, we depict the stable and unstable equilibrium point of the quadratic equations given in subsection 4.2. The parameter settings are the same as in Figure 4 apart from $\lambda_{i}^{1}$ which varies from 0 to 1 and apart from $\mu$ which is fixed to 0.7. By evaluating the eigenvalues of the Jacobian matrix (16) at the equilibrium points, we can determine the stability of these solutions. The stable equilibrium points have at least one eigenvalue that has a positive real part while the eigenvalues for the unstable equilibrium points all have a negative real part.

The fluid limit also allows for evaluating the evolution to equilibrium. The transient behaviour predicted by the fluid approximation is shown in Figures 6(a) and 6(b). We assume the same parameter settings except for the maximum number of individuals $L$ which is now equal to 50 instead of 20 . The start values are respectively $x_{r}(0)=0, x_{i}(0)=50$ and $x_{s}(t)=0$ and $x_{r}(0)=0, x_{i}(0)=0$ and $x_{s}(t)=50$ in Figures 


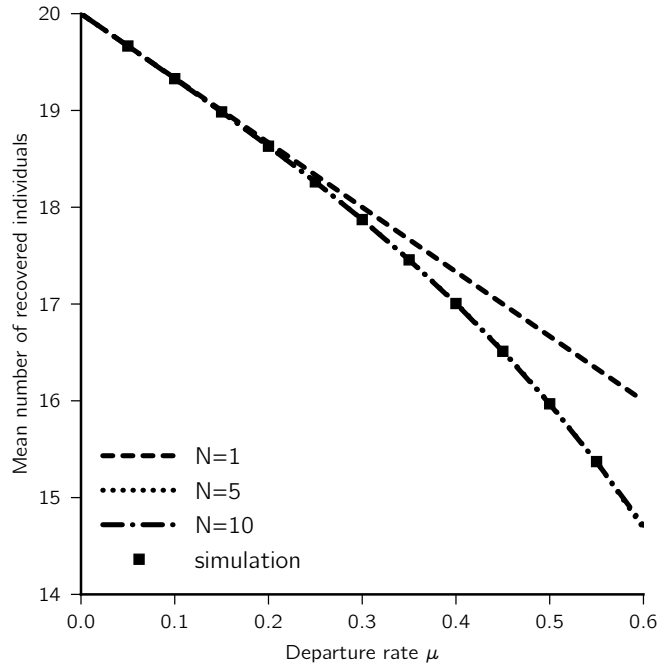

(a)

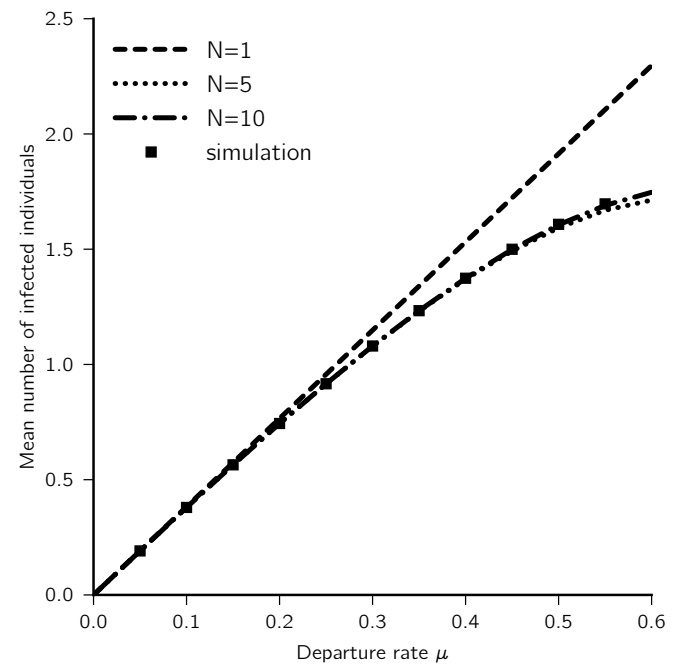

(b)

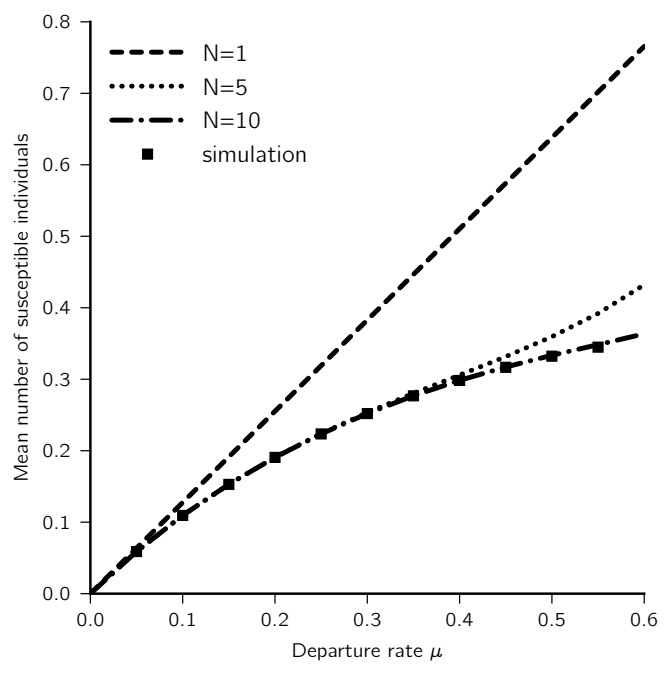

(c)

Figure 4: Mean number of recovered (a), infected (b), and susceptible (c) individuals. 


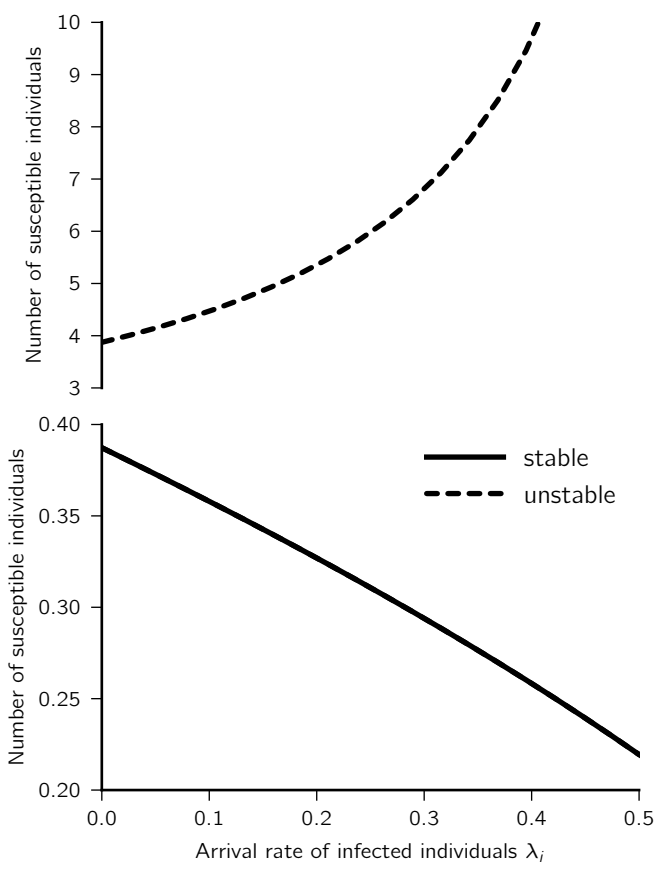

Figure 5: Stable and unstable equilibrium points of $x_{s}$ for $\lambda_{k}^{0}=3, \mu=0.7 \alpha_{s i}^{0}=\alpha_{s i}^{1}=\alpha_{s r}^{0}=\alpha_{i r}^{0}=3$.

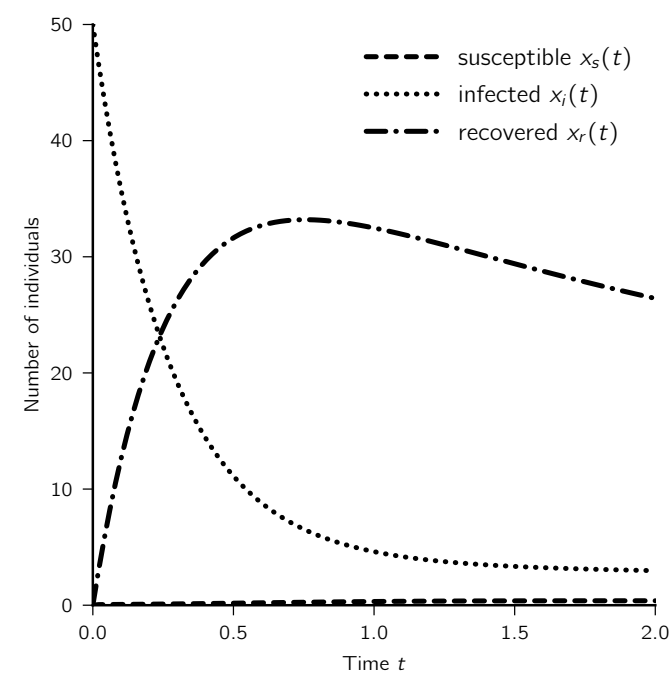

(a)

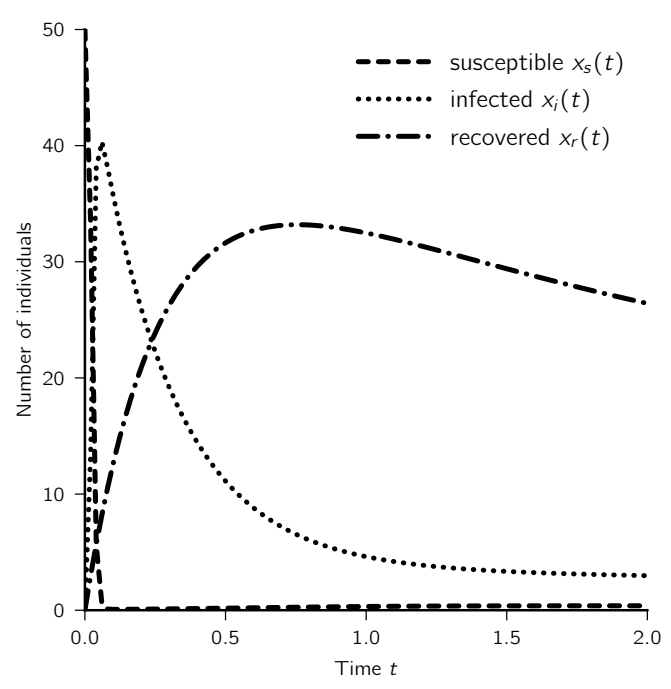

(b)

Figure 6: Fluid model for $\lambda_{k}^{0}=3, \lambda_{i}^{1}=0.1 \mu=0.7, \alpha_{s i}^{0}=\alpha_{s i}^{1}=\alpha_{s r}^{0}=\alpha_{i r}^{0}=3$ and $L=50$. 
6(a) and 6(b). As the figures show, a large number of infected or susceptible individuals quickly lead to a relative large number of recovered individuals.

Finally, we combine series- and fluid approximations. In Figure 7, we depict the mean number of susceptible, infected and recovered individuals verus $\mu$ as calculated by the Maclaurin-series expansion with $N=10$ as well as the same quantities as calculated by the equilibrium points of the fluid limit. Moreover, both approximations are compared with simulation results. The other parameters are the same as in Figure 4. This figure clearly demonstrates that both approximations are complementary: the series expansion and the fluid limit approximate well the mean number of recovered individuals for low and high values of $\mu$, respectively. It can be seen that the accuracy of the series expansion deteriorates as $\mu$ increases while the accuracy of the fluid limit deteriorates for decreasing $\mu$. In this case, combining both approximations yields a good approximation for the whole range of $\mu$ going from 0 to 1.4 .

\section{Conclusion}

In this paper, we evaluate the propagation of an opinion in a size-limited population that has a low population turnover. Furthermore, we assume that individuals can have either no opinion (S), an opinion that they want to spread (I) or an opinion that they don't want to transmit or no opinion as they become neutral or lose their interest in the topic (R). Moreover, the evaluation method at hand allows for statedependent arrival rates of the three types as well as for state-dependent rates at which an individual changes type.

To cope with the inherent state space explosion, we propose an approximative numerical algorithm for the Markovian epidemic process. In particular, a numerical algorithm is applied which calculates the first $N$ coefficients of the Maclaurin-series expansion of the steady-state probability vector. From the numerical results, we show that the series expansion approach gives us a good approximation for the opinion model in a heuristically determined region. Complementary to the series expansion approach, we derive a fluid limit of the Markov chain where the arrival rates of the three types and the population size are sent to infinity.

\section{Acknowledgements}

This research has been funded by the Interuniversity Attraction Poles Programme initiated by the Belgian Science Policy Office.

\section{References}

[1] K.E. Avrachenkov and M. Haviv. Perturbation of null spaces with application to the eigenvalue problem and generalized inverses. Linear Algebra and its Applications, 369, pp.1-25, 2003.

[2] K.E. Avrachenkov, J.A. Filar, and P.G. Howlett. Analytic Perturbation Theory and Its Applications, SIAM, 2013 


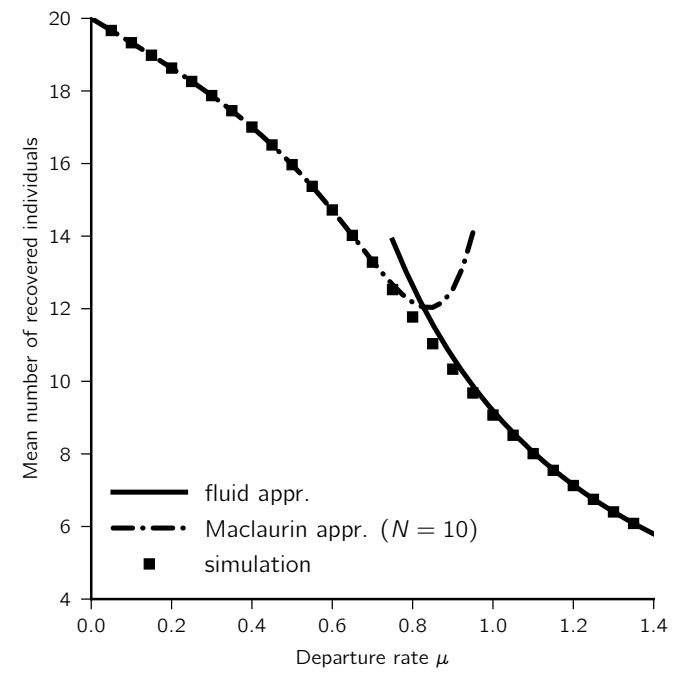

(a)

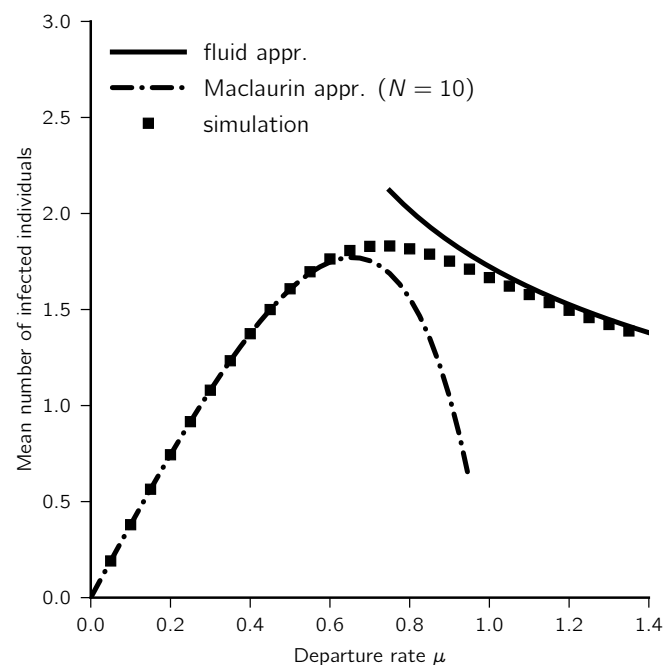

(b)

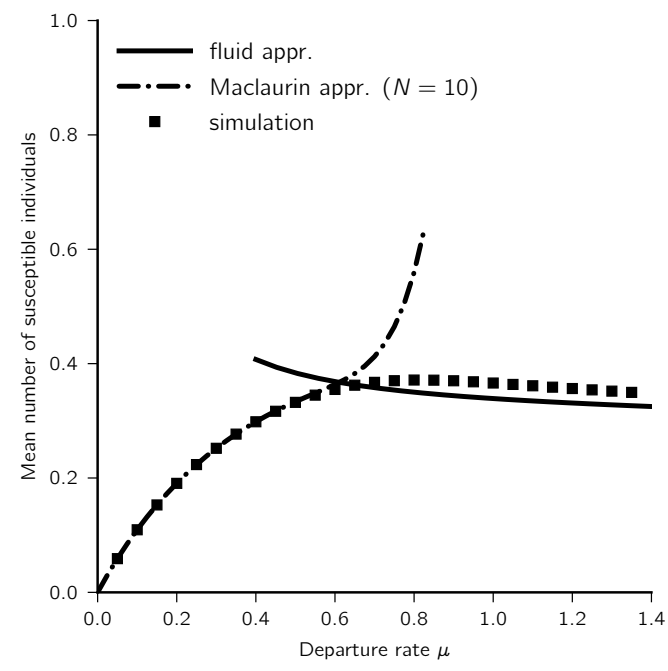

(c)

Figure 7: Mean number of recovered individuals calculated by the Maclaurin-series expansion (left) and the fluid limit (right). 
[3] E. Altman, K.E. Avrachenkov and R. Nunez-Queija. Perturbation analysis for denumerable Markov chains with application to queueing models. Advances In Applied Probability, 36(3):839-853, 2004.

[4] H. Andersson and R.M. May. Infectious Diseases of Humans. Oxford University Press, Oxford, UK, 1992.

[5] L.M.A. Bettencourt, A.Cintrón-Arias, D.I. Kaiser and C. Castillo-Chávez. The power of a good idea: Quantitative modeling of the spread of ideas from epidemiological models. Physica A: Statistical Mechanics and its Applications,364,513-536,2006.

[6] B. Błaszczyszyn, T. Rolski and V. Schmidt.Advances in Queueing: Theory, Methods and Open Problems. Chapter Lighttraffic approximations in queues and related stochastic models. CRC Press, Boca Raton, Florida, 1995.

[7] B. Błaszczyszyn. Factorial-moment expansion for stochastic systems. Stochastic Processes and their Applications. 56, 321-335, 1995.

[8] J. Blom, O. Kella, M. Mandjes, H. Thorsdottir. Markov-modulated infinite-server queues with general service times. Queueing Systems 76(4):403-424 (2014)

[9] H.G. Dai and S.P. Meyn. Stability and convergence of moments for multiclass queuing-networks via fluid limit models. IEEE Transactions On Automatic Control 40(11):1889-1904 (1995).

[10] R.W.R. Darling, J.R. Norris, Differential equation approximations for Markov chains. Probability Surveys 5 (2008), 37-79.

[11] E. De Cuypere, D. Fiems. Performance evaluation of a kitting process. Proceedings of the 18th International Conference on Analytical and Stochastic Modelling Techniques and Applications (ASMTA 2011), pp. 175-188, Venice, June 2011.

[12] E. De Cuypere, K. De Turck, S. Wittevrongel and D. Fiems. Markovian SIR model for opinion propagation. Proceedings of the 25th International Teletraffic Congress (ITC 2013), Shanghai, September 2013.

[13] K. De Turck, E. De Cuypere, S. Wittevrongel and D. Fiems. Algorithmic approach to series expansions around transient Markov chains with applications to paired queuing systems. International Conference on Performance Evaluation Methodologies and Tools (VALUETOOLS 2012), 38-44, France, October 2012.

[14] S. Ding, M. Remerova, R.D. van der Mei, B. Zwart. Fluid approximation of a call center model with redials and reconnects. Performance Evaluation 92:24-39, 2015.

[15] S.N. Ethier and T.G. Kurtz. Markov Processes: Characterization and Convergence. Wiley and Sons, Second Edition, 2005.

[16] D.S. Evans. The online advertising industry: economics, evolution and privacy. Journal of Economic Perspectives, $23(3): 37-60,2009$.

[17] P. Fan, H. Wang, P. Li, W. Li and Z. Jiang. Analysis of opinion spreading in homogeneous networks with signed relationships. Journal of statistical mechanics-theory and experiment, DOI = 10.1088/1742-5468/2012/08/P08003, August 2012.

[18] B. Heidergott, A. Hordijk, and N. Leder. Series Expansions for Continuous-Time Markov Processes. Operations Research, 58.3, 756-767, DOI=10.1287/opre.1090.0738 http://dx.doi.org/10.1287/opre.1090.0738. May 2010.

[19] H.W. Hethcote. The mathematics of infectious diseases. SIAM Review, 42, 599-653, 2000.

[20] A.L. Hill, L., D.G. Rand, M.A. Nowak and N.A. Christakis. Infectious Disease Modeling of Social Contagion in Networks. Plos computational Biology,6,11,November 2010.

[21] V. Isham, J. Kaczmarska and M. Nekovee. Spread of information and infection on finite random networks. Physical review E, 83(4), 2, April 2011.

[22] Y. Li, Z. Feng, H. Wang, S. Kong, and Ling Feng. ReTweetp: Modeling and Predicting Tweets Spread Using an Extended Susceptible-Infected-Susceptible Epidemic Model. DASFAA 2013, W. Meng et al. (Eds.), Part II, LNCS 7826, SpringerVerlag Berlin Heidelberg 2013, 454-457, 2013.

[23] G. Katriel and L. Stone. Pandemic Dynamics and the Breakdown of Herd Immunity, Plos one,5-3,2010.

[24] T. Kawamoto. A stochastic model of tweet diffusion on the Twitter network. Physica A-statistical mechanics and its applications, 392, 3470-3475, 2013. 
[25] D. Kempe, J. Kleinberg and E. Tardos. Maximizing the spread of influence through a social network. Proceedings of KDD, $137-146,2003$.

[26] W.O. Kermack and A.G. McKendrick. A Contribution to the Mathematical Theory of Epidemics. Proceedings of the Royal Society of London A, 115, 700-721, 1927.

[27] I. Kovalenko. Rare events in queueing theory, a survey. Queueing systems. 16(1): 1-49, 1994.

[28] C.J. Kuhlmana,V.S.A. Kumara and S.S. Ravib. Controlling opinion propagation in online networks. Computer Networks, 57, 10, 2121-2132, July 2013.

[29] A. Mandelbaum, W.A. Massey, and M.I. Reiman. Strong approximations for Markovian service networks. Queueing Systems 30(1-2):149-201 (1998).

[30] S. Meyn and R.L. Tweedie. Markov Chains and Stochastic Stability. 2nd edition, Cambridge University Press, 2009.

[31] Y. Moreno, M. Nekovee and A.F. Pacheco. Dynamics of rumor spreading in complex networks. Physical review E, 69, 6, 2 ,June 2004.

[32] M. Reiman and B. Simon. Open queueing systems in light traffic. Mathematics of operations research. 14(1): 26-59 (1989).

[33] P. Robert. Stochastic Networks and Queues. Springer, Berlin, 2003.

[34] P. Robert and A. Veber. A stochastic analysis of resource sharing with logarithmic weights. Annals Of Applied Probability 25(5):2626-2670, 2015.

[35] P.J. Schweitzer. Perturbation theory and finite Markov chains. Journal of Applied Probability, 5(2):401-413, 1968.

[36] A.M. Timpanaro and C.P.C. Pradot. Generalized Sznajd model for opinion propagation. Physical review E, 80, 021119, DOI: 10.1103/PhysRevE.80.021119, 2009.

[37] J. Woo, J. Son and H. Chen. An SIR model for violent topic diffusion in social media. IEEE International Conference on Intelligence and Security Informatics (ISI 2011),15-19,9-12 July 2011, Beijing, China.

[38] Z. Yang, J. Guo, J. Tang, L. Zhang and Z. Su. Understanding retweeting behaviors in social networks. In: CIKM (2010)

[39] L. Zhao, J. Wang, Y. Chen, Q. Wang, J. Cheng and H. Cui. SIHR rumor spreading model in social networks. Physica A-statistical mechanics and its applications, 391, 7, 2444-2453, April 2012. 